\title{
ALGUNOS PRINCIPIOS DEL DERECHO AMBIENTAL Y LA SENTENCIA DEL TRIBUNAL SUPERIOR DE JUSTICIA DEL PAÍS VASCO DE 17 DE OCTUBRE DE 2011
}

\author{
CRISTINA ÁlVAREZ BAQUERIZO
}

Abogada

\begin{abstract}
"Uno vence raras veces, pero alguna vez vence. Que hayamos perdido cien años antes de empezar no es razón para no tratar de ganar".

El abogado Atticus Finch a su hija Scout en la novela "Matar a un Ruiseñor", de Harper Lee.
\end{abstract}

RESUMEN: La Sentencia del Tribunal Superior de Justicia del País Vasco de 17.10.2011 ha desestimado una Demanda contencioso-administrativa interpuesta por una Coordinadora de asociaciones vecinales contra una Resolución del Gobierno Vasco concediendo una Autorización Ambiental Integrada (en adelante AAI) ${ }^{1}$ de eficacia diferida a una Refinería ${ }^{2}$. Dicha Refinería, que comenzó a operar a principio de los años setenta, esta situada en las marismas costeras del Barbadún, y en pleno casco urbano de la población de Muskiz, Vizcaya.

La Demanda interpuesta desde la Coordinadora vecinal se basó, esencialmente, en la ausencia de licencias y permisos previos (licencia de actividad y permiso de vertidos al mar), la falta de documentación explicativa de la situación fáctica preexistente respecto de las emisiones atmosféricas, la ubicación de la planta en casco urbano, la falta de pronunciamiento y de cualquier participación en el procedimiento de la administración responsable del Dominio Público Marítimo Terrestre (en adelante DPMT), las

\footnotetext{
${ }^{1}$ De las comprendidas en la Ley 16/2002 de prevención y control integrados de la contaminación (En adelante, Ley IPPC) en su carácter de instalación preexistente.

${ }^{2}$ Resolución de 6 de mayo de 2008 del Viceconsejero de Medio Ambiente, por la que se concede Autorización Ambiental Integrada de eficacia diferida para la actividad de refino de petróleo promovida por Petróleos del Norte, S.A.,(Petronor) en los términos municipales de Muskiz, Zierbena y Abanto y Ciervana (Vizcaya).
} 
afecciones a la salud de los vecinos y el hecho de que parte de los requisitos impuestos en la resolución eran mandatos legales vigentes, en algunos casos desde los años ochenta y que por ello, debían haber sido ya cumplimentados con anterioridad a la propia solicitud. ${ }^{3}$ El Tribunal Superior de Justicia del País Vasco desestimó la Demanda y hoy se encuentra en Casación ante el Tribunal Supremo.

El caso es interesante porque además de obligarnos a pensar de nuevo sobre problemas históricos e irresueltos del Derecho Ambiental como los limites de la apreciación discrecional de los Informes técnicos por parte de la Administración o la carga de la prueba, sugiere la necesidad de profundizar en como aplicar en la practica procesal lo que se han venido a llamar Principios del Derecho ambiental, en particular el principio de Precaución, y el mas recientemente formulado principio de "no Regresión del Derecho Ambiental".

\section{El tema de la ubicación}

La ubicación de la Refinería es uno de los principales problemas de este supuesto.

En primer lugar, la planta se encuentra situada en pleno casco urbano de Muskiz, de tal manera que uno de sus barrios ${ }^{4}$ es medianero con la propia Refinería, y ningún punto del casco urbano dista mas de setecientos metros de la planta. Cuando se decidió su instalación, en 1968, fue mediante Decreto 5 , por razones de "excepcional urgencia e interés público" y sin aplicar las disposiciones de la época que, a través del RAMINP ${ }^{6}$ imponían la distancia de dos mil metros a núcleo habitado de una instalación "peligrosa" como eran clasificadas las refinerías. Es decir, los barrios de Muskiz a que nos hemos referido ya estaban allí en 1968. No se trata de uno de estos casos en que la planificación urbanística permite crecimientos que acercan las poblaciones a las instalaciones en que tienen lugar actividades peligrosas. Se trata de una actividad

\footnotetext{
${ }^{3}$ Este artículo no se referirá a todos estos aspectos, sino únicamente a los referentes a ubicación, contaminación atmosférica y salud publica.

${ }^{4}$ San Julián de Muskiz.

5 Decreto 2132/68 de 14 de Septiembre por el que se resuelve el concurso...para la instalación y explotación de una Refinería de petróleos en la provincia de Vizcaya.

6 Reglamento de actividades, molestas, insalubres, nocivas y peligrosas (RAMINP), aprobado por Decreto 2114/1961, de 30 de noviembre.
} 
peligrosa que se instaló junto a las viviendas en una época en que las posibilidades de oposición vecinal eran inexistentes.

Evidentemente, esta cercanía a las viviendas vecinales se ha venido traduciendo, hace mas de cuarenta años, en todo tipo de molestias y daños, tanto derivados del funcionamiento "normal" de la planta (emisiones de contaminantes, ruidos...) como de los incidentes que a lo largo de los años y necesariamente, han tenido lugar (incendios, vertidos accidentales, explosiones, etc.).

Así pues, la Demanda planteó el RAMINP como vigente, por interpretar que sus prescripciones respecto de las distancias debían considerarse así en tanto en cuanto no hubiesen sido sustituidas por otras con el mismo nivel de protección ${ }^{7}$. Tanto el Gobierno Vasco, como demandado, como la propia empresa explotadora de la Refinería, actuando como codemandada, alegaron la inaplicabilidad del RAMINP por haber sido expresamente derogado por la Ley de Medio Ambiente del País Vasco ${ }^{8}$. La Sentencia se mostró de acuerdo con estas alegaciones, señalando que el RAMINP era inaplicable y no seria tomado en consideración, puesto que dicha Ley de Medio Ambiente del País Vasco establece un régimen similar en sus artículos 55 y siguientes ${ }^{9}$.

Desde luego, cuando desde la representación letrada de los vecinos planteamos la vigencia y necesaria consideración del RAMINP no lo hacíamos pensando en una "migración" de la planta. Lo hacíamos pensando en que considerar la cercanía a las viviendas de la población y la inobservancia del RAMIP que tuvo lugar en su día justificaba un especial celo en el establecimiento de las condiciones de funcionamiento de la Refinería, sobre todo, en lo referente a contaminación atmosférica, afecciones a la salud y planes de emergencia exteriores.

En segundo lugar, la Refinería se encuentra situada en Dominio Publico Marítimo Terrestre, sobre una zona de marismas. Viene ocupando dicha zona en virtud de un conjunto de tres concesiones otorgadas en 1982, cuya vigencia era treinta años, por lo que su vencimiento estaba previsto para este año de 2012. Ante tal situación, la Demanda reprochó al Gobierno Vasco que la administración competente de la tutela del DPMT no se hubiese pronunciado al respecto de la concesión de la AAI. En esta

\footnotetext{
${ }^{7}$ Ver Jurisprudencia al respecto en el apartado 5 de este Articulo.

${ }^{8}$ Ley 3/1998, de 27 de febrero, General de Protección del Medio Ambiente del País Vasco, en la que no se hace referencia alguna a distancias o emplazamientos.

${ }^{9}$ Fundamento de Derecho Cuarto, A, pagina 14 de la Sentencia
} 
ocasión, las contrapartes alegaron que la Ley IPPC establece ${ }^{10}$ que “... La autorización ambiental integrada se otorgará sin perjuicio de las autorizaciones o concesiones que deban exigirse para la ocupación o utilización del dominio público, de conformidad con lo establecido en... la Ley 22/1988, de 28 de julio, de Costas, y demás normativa que resulte de aplicación".

La expresión "sin perjuicio" fue así comprendida como la innecesariedad de contar con pronunciamiento alguno de las autoridades de costas en el marco del procedimiento autorizatorio que es la AAI. Tal pronunciamiento debería producirse al margen del procedimiento autorizatorio, al parecer. Este sinsentido fáctico y jurídico que no acertábamos a explicarnos en los primeros momentos quedó lamentablemente claro en Diciembre de 2010, cuando el Parlamento del Reino, mediante un pacto de ultima hora entre PP, PSOE y PNV incorporó a la Ley de Sostenibilidad una Disposición adicional mediante la cual las concesiones de uso de DPMT otorgadas para instalaciones de Refino de petróleo podían ser prorrogadas por el mismo tiempo que tuviese la concesión inicial, en contra de lo establecido por la Ley de Costas. ${ }^{11}$

\section{El tema de la Contaminación atmosférica}

La Ley IPPC establece ${ }^{12}$ que junto con la solicitud de AAI, los promotores han de adjuntar un "Proyecto básico" que incluya, al menos, los siguientes aspectos: “...Descripción detallada de: Fuentes generadoras de las emisiones de la instalación... Tipo y cantidad de las emisiones previsibles de la instalación al aire...”.

Respecto a la contaminación atmosférica emitida por la Refinería, son precisas algunas explicaciones previas. Hace ya décadas que el esquema de la legislación sobre contaminación atmosférica implica tres elementos: un marco general legislativo, una serie de normas derivadas respecto a los valores limites y umbrales de seguridad de ciertos contaminantes, y una serie de normas especificas sobre sistemas de control y muestreo.

\footnotetext{
${ }^{10}$ Articulo 11,3) de dicho texto legal.

${ }^{11}$ Ley 2/2011, de 4 de marzo, de Economía Sostenible., que en su Disposición final vigésima novena. establece una "Modificación de la Ley 34/1998, de 7 de octubre, del Sector de Hidrocarburos",

${ }^{12}$ Articulo 16,1,a) de dicho texto legal.
} 
Mientras las normas de marco general resultan normas comprensibles, el desarrollo de las mismas a través de las normas de contaminantes concretos y de sistemas de control ofrece dificultades crecientes para ser comprendido. Se trata de normas muy técnicas, cuya comprensión requiere de conocimientos previos de química y de ingeniería. ${ }^{13}$ Podría decirse que a medida que se han venido produciendo avances técnicos, la redacción de estas normas ha pasado de una cierta dificultad a la ininteligibilidad casi absoluta.

Además, a este aspecto se añade otro, y es que en el caso de emisiones de grandes plantas, y desde $1976^{14}$, las empresas son quienes efectúan sus propias mediciones, teniendo el deber de comunicarlas a las administraciones competentes, que proceden a incorporarlas a sus estadísticas tal cual las reciben.

A estos dos elementos, hay que añadir aun un tercer antecedente; la Refinería, a la fecha de la Resolución recurrida (18 de noviembre de 2008), no tenia instalados los sistemas que permitieran la toma de muestras de la gran mayoría de sus focos emisores. Este hecho no fue negado de contrario en ningún momento, puesto que a lo largo del procedimiento y antes, había quedado de manifiesto en varias ocasiones. ${ }^{15}$

Teniendo en cuenta estos tres factores, nos encontramos con que en el procedimiento de solicitud de la AAI, la empresa no aporta realmente información sobre sus emisiones atmosféricas a aquella fecha. Aporta, dentro de la memoria del Proyecto, un Documento $^{16}$ titulado "Emisiones atmosféricas" conteniendo principalmente datos de inmisión, no de emisión. En ningún caso, los datos exigibles sobre fuentes generadoras de las emisiones de la instalación... Tipo y cantidad de las emisiones previsibles de la instalación al aire...". También manifiesta la promotora, y obraba en el expediente, que los escasos datos de emisión proporcionados — que como hemos dicho, no describían todos los focos ni todos los contaminantes_-, eran una "estimación, realizada de acuerdo a modelos informáticos".

La Demanda puso un especial énfasis en esta situación, señalando que sin contar con datos fiables, completos y reales sobre las emisiones de la Planta, no estaba la

\footnotetext{
${ }^{13}$ Para este caso, en particular, RD 646/1991, RD 1800/1995, RD 1073/2002 y RD 430/2004.

14 Orden de 18 de octubre de 1976 sobre prevención y corrección de la contaminación industrial de la atmósfera. (Vigente hasta el 30 de enero de 2011).

${ }^{15}$ De hecho, la Resolución recurrida establecía entre su condicionado, el establecer estos sistemas.

${ }^{16}$ Capitulo IV de la Memoria.
} 
administración actuante en condiciones de emitir Resolución autorizatoria alguna. No se solicitó prueba pericial, teniendo en cuenta varias razones: sobre todo, y en primer lugar, porque en pura lógica, entendíamos que una ausencia no puede ser objeto de prueba mas allá de su mera constatación. En segundo lugar, porque el Gobierno vasco nunca elaboró informe técnico alguno al respecto, que tuviésemos que contradecir o matizar. En tercer lugar, porque en el procedimiento figuraban dos dictámenes procedentes de sendos expertos en contaminación industrial que ponían de manifiesto la carencia de datos reales sobre emisiones ${ }^{17}$, informes que no fueron objeto de contradicción, tacha o reproche técnico alguno por ninguna de las partes. Y en cuarto lugar, porque entendíamos que en estas condiciones, la aplicación del principio de Precaución debía hacer innecesaria la aportación de prueba alguna por nuestra parte, aspecto al que nos referiremos con más detalle mas adelante.

El Gobierno Vasco mantuvo a lo largo del pleito que la información aportada le resultaba suficiente. Y la empresa, que el criterio técnico de la administración, aceptando la documentación presentada sobre el cumplimiento de los estándares de emisiones atmosféricas era el que debía primar.

La Sentencia dice que correspondía a la demandante la obligación de probar que información era la que no concurría en el expediente. Dice también que es carga de prueba para la demandante probar, mediante pericial, la ausencia alegada. Señala, además, que el Tribunal carece de conocimientos técnicos para interpretar correctamente las normas alegadas;

"En definitiva existe una notoria carencia de prueba pericial que dote al tribunal de los conocimientos específicos para determinar la existencia o no de incumplimientos como los alegados. En estas condiciones no es posible destruir la presunción de acierto del órgano técnico ambiental que ha avalado la suficiencia de la documentación presentada y la adecuación de su contenido".

Además, la Sentencia señalaba que ${ }^{18}$ “el Tribunal carece de conocimientos técnicos que le permitan concluir que las medidas que trascribe... sean las aplicables, pues de la lectura del Articulo 2 de la norma no puede concluirse eso sin una prueba pericial". Es

\footnotetext{
${ }^{17}$ Emitidos en el trámite de alegaciones, y encargados respectivamente por los dos Ayuntamientos en cuyo término municipal se encuentra la Refinería; Muskiz y Abanto y Ciervana.

${ }^{18}$ Fundamento Sexto, pagina 23 de la Sentencia.
} 
decir, que el Tribunal dice no poder comprender ni aplicar la norma con una mera lectura, sin la ayuda de una pericial.

\section{El tema de las afecciones a la salud.}

La misma Ley IPPC que venimos citando, obliga, en su Artículo 7, a que para la determinación en la autorización ambiental integrada de los valores límite de emisión, se deberá tener en cuenta: “...e) La incidencia de las emisiones en la salud humana potencialmente afectada".

En el procedimiento que estamos comentando, esta obligación se dijo cubrir mediante un documento emitido por la Unidad de Sanidad Ambiental de Vizcaya, ${ }^{19}$ de catorce líneas de extensión en las que básicamente se viene a decir que respecto del expediente en tramitación, "no procede hacer observación alguna al mismo".

Se daba la circunstancia, por otra parte, de que a la fecha de redacción de la Demanda, el Gobierno Vasco estaba ultimando un trabajo tendente a establecer las causas de mortalidad por áreas en la Comunidad $\mathrm{Vasca}^{20}$. Los datos para los dos municipios afectados ya estaban ultimados, y en ellos se observa que la mortalidad en el municipio de Muskiz es significativamente superior a la del conjunto de la población vasca, tanto para los varones (exceso de mortalidad superior al 20\%), como para las mujeres (entre un 10 y un $20 \%$ mayor). Al analizar las causas específicas de muerte en este municipio se constata un "exceso de mortalidad por tumores malignos y en varones y mujeres, por enfermedades vasculares cerebrales en las mujeres y por enfermedad pulmonar obstructiva crónica en los varones ${ }^{21}$. Aparte de esto, llama la atención la mortalidad significativamente elevada de las niñas de 0-14 años en el municipio adyacente de Zierbana, que no se observa en casi ningún otro municipio de la Comunidad Autónoma Vasca".

\footnotetext{
${ }^{19}$ Pagina 737 del expediente

${ }^{20}$ I. MONTOYA, S. ESNAOLA, R. RUIZ, C. AUDICANA, E. ALDASORO, M. CALVO, B. IBÁÑEZ, "Atlas de mortalidad en áreas pequeñas en el País Vasco. Análisis geográfico de la mortalidad por sexo y grupos de edad en las secciones censales del País Vasco (Proyecto MEDEA, País Vasco)", GUDO 3, III Jornadas Científicas de las Sociedades Españolas de Epidemiología y Biometría, Valencia, 22 y 23 de Junio de 2006.

${ }^{21}$ I. MONTOYA, S. ESNAOLA, R. RUIZ, C. AUDICANA, E. ALDASORO, M. CALVO, B. IBÁÑEZ, Patrón geográfico de las principales causas de mortalidad en las secciones censales del País Vasco, XXV Reunión Científica de la Sociedad Española de Epidemiología, 2007.
} 
Consecuentemente a todo lo expresado mas arriba, la Demanda alegó que no se habían valorado las incidencias de la actividad sobre la Salud humana. Además, se aportó como prueba documental una copia del citado Informe y se solicitó la testifical de la Directora General de Salud Publica del Gobierno vasco. En su declaración, dicha señora, preguntada por si "contaban en su Departamento con información suficiente para valorar las afecciones a la salud de la población del proyecto", respondió que "contaban con la información que les proporcionaba la Dirección General de Calidad Ambiental". El propio Tribunal, aplicando el artículo 376 de la LEC, entendió que la respuesta era negativa. Es decir, se reconocía que el Gobierno Vasco no contaba con información suficiente para valorar las afecciones de la Refinería en la salud de la población de su entorno.

El Gobierno Vasco y la empresa mantuvieron, al respecto, la misma posición, alegando que si había tenido lugar dicha valoración, aunque de manera indirecta, puesto que entendían que las emisiones estaban por debajo de los niveles legales establecidos. Y que la legislación, en si misma, es una manera de velar por la salud de la población. El Gobierno Vasco, además, y sobre el Informe citado, señaló por un lado, que no estaba terminado, y por otro, que no se podía demostrar relación de causalidad entre la alta morbilidad de la zona con la presencia de la Refinería ${ }^{22}$.

La Sentencia señaló finalmente que “... la Sala carece de elementos de juicio suficientes para determinar que no se ha ponderado la incidencia de las emisiones en la salud humana afectada puesto que los datos presentados no prueban nada a este respecto".

\section{Hablábamos de principios: el principio de Precaución}

Los Principios del Derecho, nos enseñaban en la Facultad, cumplen una función de interpretación, de aplicación de la norma y de integración, porque cubren vacíos legislativos, actúan como diques de contención para mantener la pureza del sistema, sirven para renovar la normativa obsoleta y como mecanismo de innovación.

\footnotetext{
22 Fuera de sede judicial, en reuniones y entrevistas que tuvieron lugar los meses precedentes, la Dirección de Salud publica había afirmado que la relación causa/efecto no estaba clara, ya que aunque no existían mas empresas contaminantes en la zona, la población es de nivel socioeconómico mas bajo que el resto de la Comunidad, por lo que el tabaquismo y la mala alimentación son también más frecuentes, y estos factores podían ser igualmente responsables de las altas tasas de mortalidad.
} 
Y se ha afirmado que, de entre los principios del Derecho ambiental, el que mas le diferencia de las demás disciplinas jurídicas clásicas es precisamente el de Precaución ${ }^{23}$. Ha sido formulado mediante la expresión de que "[1]a falta de certeza científica no debe utilizarse como razón para posponer medidas destinadas a prevenir el deterioro del medio ambiente". La Declaración de Río de Janeiro $^{24}$ se refiere al empleando el concepto de "riesgo": "Cuando haya amenaza de daño grave irreversible, no deberá utilizarse la falta de total certidumbre científica, como razón para posponer medidas de protección ambiental". Con origen en el ordenamiento jurídico alemán ${ }^{25}$, ha sido recogido de manera expresa en multitud de textos jurídicos de Derecho Internacional ${ }^{26}$.

Así pues tenemos que teóricamente, el principio de Precaución opera sobre el concepto del riesgo. Pero... ¿Cuáles son sus los efectos sobre las políticas publicas, cuál es el contenido del principio y sobre todo, cuáles son los presupuestos de aplicación?

Refiriéndonos a este ultimo aspecto, en materia procesal, y por ende, en el marco de procedimientos como el que nos ocupa, el Principio de Precaución determinaría que la carga de la prueba dejase de ser estática (“debe probar aquel que alega,”), para pasar a ser dinámica, obligando a la carga de la prueba a quien se encuentre en mejores condiciones técnicas y económicas para probar. ${ }^{27}$

En otros ordenamientos jurídicos, la doctrina ha venido intentando construir teorías que flexibilicen la prueba. Y en materia de "daños" se han abierto algunos caminos. Así, en la Ley alemana de Medio Ambiente ${ }^{28}$ se establece una presunción de causalidad consistente en que "si una instalación, dadas las circunstancias del caso particular, es apropiada para originar el "daño" producido, se presume que el mismo ha sido causado por esa instalación".

\footnotetext{
${ }^{23}$ A veces también denominado de "Cautela", como en el Articulo 191 del TFUE

${ }^{24}$ Principio 15 de la Declaración de Río de Janeiro

${ }^{25}$ Ley alemana del Medio Ambiente, 1971

${ }^{26}$ Primera y la Tercera Conferencia Internacional del Mar del Norte, entre 1987 y 1990, Convenio de Bamako, África, sobre Movimientos Transfronterizos de Desechos Peligrosos 1991, Convenios relativos a la protección del Mar Báltico, Preámbulo de la Convención sobre Diversidad Biológica, artículo 3.3 de la Convención Marco del Cambio Climático Protocolo de Cartagena sobre Bioseguridad, aprobado en Montreal en 2000. También Artículo 5 de la Carta del Medio Ambiente de la Republica Francesa.

${ }^{27}$ Los principios y reglas del Derecho ambiental, Néstor Cafferatta, 2010

${ }^{28}$ De 10 de diciembre de 1990
} 
Pero no son los "daños", (que requieren prueba de carácter tan especializado y técnico a la que la mayoría de las veces en el procedimiento español, no se podrá hacer frente, desde entidades cívicas, a los costes de los expertos), sino los "riesgos" el verdadero campo de aplicación del principio de Precaución. Y al respecto, nos encontramos con una situación monolítica en la cual, y en particular, en lo que se refiere a los avances en el control jurisdiccional de las decisiones administrativas, no se atisban aun fisuras a través de las que este principio se emplee realmente en el Reino de España.

Según la Jurisprudencia comunitaria, ${ }^{29}$ se identifican dos elementos o condiciones como necesarios para dar lugar a la aplicación del principio de Precaución; son en primer lugar, la identificación de la existencia de un peligro y en segundo lugar, su gravedad.

Deben cumplirse ambas condiciones aunque no sea imprescindible que se demuestre plenamente la realidad y gravedad de tales riesgos. En caso contrario, es decir, si no se exigieran al menos indicios razonablemente suficientes de la existencia de un peligro o se aplicara el principio de precaución cuando el riesgo es seguramente mínimo (despreciable), se banalizaría su eficacia jurídica con efectos imprevisibles.

¿Es un peligro real para la vida y la seguridad de las personas que una Refinería se encuentre instalada en pleno casco urbano? ¿Es grave, el riesgo? ¿Que tipo de prueba puede ofrecerse al respecto? ¿Debemos aceptar como ciudadanos de un estado democrático y como profesionales del Derecho que tales extremos deban ser objeto de prueba? ¿Bastaría a nuestros propósitos de tutela sobre la calidad ambiental de la población que, en aras al principio de Precaución, y en casos como este se invirtiese la carga de la prueba?

En mi opinión, la inversión ocasional de la carga de la prueba en ciertos supuestos ambientales sería sin duda precisa, pero no solucionaría las dificultades que encuentra la aplicación práctica del Principio de Precaución.

Las decisiones de las administraciones públicas autorizando actividades de riesgo, o de incertidumbre en cuanto a riesgo, tienen un carácter marcadamente impositivo. En cuestiones ambientales la Administración impone en general sus decisiones a la sociedad en un marco de casi inexistente participación publica y como si no hubiese opciones. Además, actúa en un escenario en el que quien promueve y explota una

\footnotetext{
${ }^{29}$ Sentencia TJCE Reino Unido/Comisión de 5 de mayo de 1998, asunto C-180/96 relativo a las medidas de protección a los consumidores aplicadas por la Comisión de la UE tras la crisis de las EEB ("vacas locas").
} 
industria contaminante, en realidad, está repartiendo los riesgos pero no los beneficios de su actividad. Añadamos a esto que la Administración no cuenta con recursos, ni plazos suficientes para conocer adecuadamente la realidad científico-técnica. De ahí, por ejemplo, la dejación de sus competencias en manos de "entidades colaboradoras" que controlan la contaminación atmosférica de los grandes focos ${ }^{30}$. El resultado es que las administraciones públicas no están cumpliendo con su función de tutelar los derechos constitucionales a un medio ambiente digno. Como mucho logran canalizar la actividad industrial o económica dentro de una perspectiva única e incuestionada y el resultado no es el bienestar ciudadano.

Si deseamos que las decisiones sobre riesgos tengan algo que ver con la realidad y al mismo tiempo sean democráticamente legítimas, se requiere claramente de la mejora de las relaciones entre el ordenamiento jurídico y el ámbito científico-técnico.

En el plano de la técnica normativa los problemas mas evidentes se dan en la remisión reglamentaria al "ordenamiento de la técnica", a través de la cual se intenta legitimar democráticamente el traspaso del poder normativo al científico. En la practica, esta remisión, cada vez mas amplia y frecuente - como en el caso citado de las normas sobre contaminación atmosféricas, o en el mas fragante de las "mejores técnicas disponibles"-, esta suponiendo de facto una desregularización. El Derecho, incapaz de regular una realidad que no comprende y que evoluciona a una velocidad muy superior a las posibilidades de los procedimientos normativos existentes, idea mecanismos para permitir que dicha realidad se regule a sí misma ${ }^{31}$.

En el plano procesal, los aspectos técnicos y científicos están limitando extraordinariamente la función del poder judicial, que sigue otorgando, en los asuntos ambientales, la máxima importancia a la prueba, no ya de los hechos, sino también de los riesgos, como hemos visto en el caso comentado. No aplicándose el principio de Precaución, las consecuencias jurídicas de un pleito se asignan solo de acuerdo a los resultados de la prueba. En ámbitos de alta complejidad técnica como el que estamos comentando, el Tribunal, que desconoce completamente la realidad sobre la que juzga, se limita a exigir la intervención de peritos cuando ni los problemas de la disponibilidad de estos ni de su imparcialidad real han sido abordados ni resueltos. La actuación de los

\footnotetext{
${ }^{30}$ Véase nota 14.

${ }^{31}$ A.E. EMBID TELLO, Retos jurídicos de la radiación no ionizante, Tesis doctoral, 2009.
} 
Tribunales en asuntos ambientales, asignando sin fisuras la carga de la prueba al demandante, están convirtiendo en "no peligroso" todo aquello de cuya peligrosidad se duda. Justo lo contrario de lo que se pretendía con el principio de Precaución.

En materia ambiental ${ }^{32}$, esta rigidez, propia de otros tiempos, lleva mucho tratando de ser disuelta mediante la figura de la inversión de la carga de la prueba, que permite al juez, en determinados casos, decidir la asignación de dicha prueba al demandado, pero esta solución no resulta convincente, pues es el mismo concepto de "prueba" y su naturaleza, los que resultan anticuados, no su utilización. El problema quedaría dentro del sistema jurídico si no fuera porque la importancia asignada a la prueba pericial es tal, que sigue siendo ésta la que resuelve el litigio en ámbitos donde dicha prueba "no es real".

La consecuencia de este estado de cosas es que, en la actualidad, las víctimas ambientales del desarrollo tecnológico están, al mismo tiempo, sufragándolo, y no retienen ni la más mínima capacidad de decisión respecto a qué tecnologías se implementan y cuáles no. Por otra parte, los productores no tienen incentivo alguno para investigar sobre los riesgos de sus tecnologías (ya que no van a ser sancionados por riesgos que en su día no se conocían, se van a esforzar lógicamente por "no conocer", opción acorde con el ánimo de lucro que guía su actividad). Los daños a la salud y al medio ambiente que causan estas tecnologías se experimentan así continuamente en una suerte de laboratorio mundial aportando resultados gratuitos a su causante que le permiten corregir los defectos de su producto de cara a la posteridad. Y mientras tanto, la población permanece en un estado de incompetencia total sobre la determinación de los riesgos a los que se la somete.

\section{Y seguimos con los principios: la No Regresión del Derecho Ambiental}

En la ultima publicación del Observatorio de políticas ambientales, su Director ${ }^{33}$ señala que, en la actualidad, y "a poco que se profundice, las políticas de medio ambiente muestran su debilidad, que deriva del carácter secundario en el que han terminado siendo situadas por un sistema político y económico que sigue contemplando el

\footnotetext{
${ }^{32}$ Citando nuevamente al profesor Antonio Eduardo Embid Tello

${ }^{33}$ F. LÓPEZ RAMÓN, "Introducción general: Regresiones del Derecho Ambiental”, Observatorio de Politicas Ambientales 2011, Pamplona, Thomson-Aranzadi, 2011.
} 
territorio como objeto de conquista, dominio y colonización. La legislación ambiental tiende a ser internalizada como un elemento más, determinante de los costes de producción, del funcionamiento del mercado o de las políticas públicas. Así, esa legislación ha permitido que las respectivas responsabilidades hayan ido limitándose: las empresariales mediante el cumplimiento de protocolos y normas técnicas, las financieras a través del juego de intercambios sobre los derechos a contaminar y las políticas por medio de la incorporación de las directivas europeas y la observancia de los mecanismos de evaluación objetiva"

Las anteriores manifestaciones vienen al caso en el contexto de reflexión del autor respecto del principio de no regresión del Derecho ambiental. El principio de no regresión ${ }^{34}$ puede expresarse en un aserto similar a "no disminuir el patrimonio natural a transmitir a las generaciones futuras como garantía de progreso, disminuyendo el alcance de las normas jurídicas dedicadas a su conservación"35.

Cuando se realiza una mirada retrospectiva sobre la evolución del Derecho Ambiental en su reciente historia en el plano internacional, estatal y autonómico, se llega fácilmente a dos conclusiones: la primera, que los logros se obtuvieron en un tiempo relativamente breve; la segunda, que el nivel de resultados alcanzados, al menos en términos de producción normativa, fue importantísimo. Demasiado bueno para durar. Consecuentemente, en la actualidad, como acertadamente señalan los profesores citados, se perciben intentos de hacer retroceder el Derecho Ambiental, que comienza a acusar la inestabilidad derivada de la profunda lucha de intereses que subyace en su trasfondo.

Este es el caso, desde nuestro punto de vista, del Reglamento de Actividades, Molestas, Insalubres, Nocivas y Peligrosas (RAMINP) ${ }^{36}$, El RAMINP contenía desde el inicio algunas determinaciones de prevención de la contaminación, como las relativas a la depuración de aguas residuales y a los vertidos (Art. 16 y 17), o las relativas a la contaminación atmosférica por humos, gases y olores (Art. 11 y 18). A medida que

\footnotetext{
${ }^{34}$ Formulado y defendido en 2010 por el jurista francés Michel Prieur, uno de los pioneros del Derecho ambiental europeo

35 M. PRIEUR, "De l'urgente nécessité de reconnaître le principe de non régression en droit de l'environnement", Ponencia presentada en la III Reunión Mundial de Juristas y de Asociaciones del Derecho Ambiental, Rio+20: ¿quelle ambition pour l'environement?, realizada entre los días 29 de septiembre y 1 de octubre de 2011 en la Universidad de Limoges, Francia.

${ }^{36}$ Ver nota 6.
} 
fueron reglamentándose otras exigencias y controles ambientales, la licencia de actividad clasificada se ocupaba de garantizar el cumplimiento de las mismas. Por ejemplo, el control del cumplimiento de los niveles de emisión de contaminantes atmosféricos se efectuaba a través de la licencia de actividades. Y especialmente, para las industrias fabriles peligrosas o insalubres se ordenaba su alejamiento de 2.000 metros de los núcleos más próximos de población.

Se trataba de una norma sencilla, de fácil comprensión y de una técnica jurídica muy sólida que le ha permitido mantener una vigencia prolongada, si bien polémica en los últimos años. Se ha dicho que nació con una inspiración sanitaria y urbanística ${ }^{37}$ - lo que no parece negativo en sí mismo, dado el abandono que de los aspectos de salud publica viene haciendo gala el actual Derecho ambiental-y que supo adaptarse a los nuevos requerimientos ambientales. En la práctica, se erigió en la principal norma de control sanitario y ambiental de las actividades molestas, insalubres, nocivas y peligrosas. Un control que era realmente integrado porque controlaba todas las formas de contaminación de la actividad ${ }^{38}$.

A partir de los años ochenta, la mayoría de las CCAA fueron dictando legislación propia sobre actividades clasificadas con la que, en principio, desplazaban la aplicación del RAMINP. La legislación autonómica no podía derogar una norma estatal, así que el efecto que se produjo fue el del desplazamiento de la aplicabilidad de las normas estatales, es decir, se consideraba que seguía vigente como derecho supletorio. De hecho, no fue derogado ni siquiera por la propia Ley IPPC.

La STS de 1 de abril de 2004, relativa a Castilla y León, fue la primera ${ }^{39}$ en examinar la aplicabilidad del Articulo 4 del RAMINP —el relativo a las distancias - en los casos en que las CCAA no hubieran establecido normas al respecto en su legislación de actividades clasificadas. La cuestión se centraba en la exigencia de la norma de la distancia mínima que preveía el RAMINP y que no se había incorporado a la legislación castellano-leonesa. Esto fue lo que entendió el Tribunal:

\footnotetext{
${ }^{37}$ J.F. ALENZA GARCÍA, "El fin de la polémica aplicabilidad en Navarra del reglamento de actividades molestas, insalubres, nocivas y peligrosas. Comentario a la STSJ de Navarra de 2 de Octubre de 2008, sala de lo contencioso-administrativo".

38 J. ESTEVE PARDO, "El modelo de intervención integral. Conceptos, objetivos y operatividad", Derecho del medio ambiente y Administración local, 2a . ed., Fundación Democracia y Gobierno Local, Barcelona, 2006.

39 Posteriormente se dictaron las SSTS de 19 de julio de 2004 y de 27 de junio de 2007 relativas a Navarra.
} 


\begin{abstract}
"la norma de aquel articulo 4 referida a la distancia mínima exigible engarza directamente con los títulos competenciales relativos a la protección del medio ambiente, en el que las Comunidades Autónomas tienen atribuida la facultad de establecer normas adicionales de protección (articulo 149.1.23a de la Constitución), con la consecuencia, en lo que ahora importa, de que el apartamiento de aquella norma en el territorio de una Comunidad Autónoma exigirá que la normativa propia de esta la haya sustituido, sin duda alguna, por otra cuya potencialidad protectora no sea menor, lo cual no se aprecia en aquella Ley autonómica"
\end{abstract}

Pese a la reiteración de esta interpretación en tres Sentencias hasta la fecha, y como vemos en la Sentencia comentada en este Articulo, los Tribunales continúan sin aplicarla. Aceptan la Regresión del Derecho Ambiental. Ninguna disposición de carácter ambiental ha vuelto a utilizar una regla sobre las distancias, ni ha hecho descansar la autorización de las instalaciones contaminantes en su alejamiento de los núcleos de población agrupada. Se han impuesto nuevas técnicas (evaluación anticipada del impacto ambiental, control y prevención integrados de la contaminación, uso de las mejores tecnologías disponibles, imposición de medidas correctoras y posibilidad de revisión de las mismas, autocontroles, etc.).

Técnicas más complejas, técnicas en que la carga de la prueba exigida sistemáticamente a los demandantes dificulta extremadamente la tutela judicial — no eran precisos peritos para comprobar si una instalación peligrosa estaba o no al menos a dos kilómetros de un núcleo de población-, técnicas, en fin, en las que reina la discrecionalidad de la administración en su valoración de aspectos técnicos a contener en las Resoluciones que dicta. Normas más complejas, normas cuyas posibilidades de control real tanto por la población como por los Tribunales, son más escasas.

\title{
6. Lección aprendida
}

La Sentencia comentada nos fue notificada en noviembre. Luego a la fecha de redacción de este trabajo, han transcurrido menos de tres meses. Poco tiempo para digerir el polvo mordido y para extraer todas las lecciones que con el tiempo, enfocaremos mejor. 
La primera de las conclusiones a enunciar es que en estos momentos sociales e históricos es preciso un esfuerzo imaginativo y de perspectiva a la hora de enfocar, en los Tribunales, los temas de Derecho Ambiental. Esmerándonos en el estudio del caso y de la jurisprudencia aplicable, desde luego, pero insistiendo, a la vez, en lograr para los casos ambientales una visión diferente. Insistiendo más, y no menos, en la aplicación practica de los principios que la Doctrina mas avanzada en estas cuestiones nos va señalando. Aprovechando el acervo de investigación ya realizada para que cada nuevo caso formulado ante un Tribunal implique una nueva oportunidad para pulir y mejorar esos principios.

Si en este caso los vecinos hubieran decidido aportar pruebas periciales y hubiesen logrado reunir los, como mínimo veinte o veinticinco mil euros que nos hubiese costado una modesta pericial, hubiésemos seguramente ganado este caso. Puede que en el futuro lo hagamos, si tenemos la oportunidad, en otros supuestos parecidos, ya que la oposición social a la Refinería en cuestión no ha hecho más que empezar. Pero eso no seria tampoco particularmente útil. Cuando se trabaja en Derecho Ambiental, la perspectiva del "cliente" que se suele tener cuando se ejerce la abogacía en otras ramas del ordenamiento, se ensancha y se abre, y en ese escenario estamos todos los seres vivos, y buena parte de nuestras expectativas de bienestar. Con ese escenario en mente nuestra tarea tiene que tener una perspectiva de medio y largo plazo, y estar cargada de paciencia. Cada caso ha de procurar su buen desenlace tanto como abrir caminos para otros casos y situaciones.

Bajando mucho más al terreno, es imprescindible estrechar lazos entre profesionales de la abogacía, estudiosos de las ciencias jurídicas, tribunales, técnicos y expertos ambientales. Si se avanza en esa relación, y se insiste, sobre todo, en la participación ciudadana, nuestras opciones van a multiplicarse. En resumen: los abogados ambientalistas que pleiteamos en favor de los colectivos cívicos necesitamos el apoyo de los expertos y de las universidades en que estos desempeñan sus funciones. Y estos últimos necesitan "tocar" tierra para ser más eficaces en sus asertos, puliéndolos en la experiencia cotidiana de la actividad judicial. A ello. 